\title{
A XENON SOLAR NEUTRINO DETECTOR
}

\author{
A.Sh. Georgadze*, H.V. Klapdor-Kleingrothaus ${ }^{\dagger}$, H. Päs ${ }^{\dagger}$ \\ and Yu.G. Zdesenko* \\ *Institute for Nuclear Research, 252028, Kiev, Ukraine \\ $\dagger$ Max-Planck-Institut für Kernphysik, \\ P.O. Box 103980, D-69029 Heidelberg, Germany
}

\begin{abstract}
The neutrino capture by ${ }^{131} \mathrm{Xe}$ with a threshold at $352 \mathrm{keV}$ as reaction to detect solar neutrinos is examined. The most important feature of this process is its high sensitivity to beryllium neutrinos, which contribute approximately $40 \%$ to the total capture rate (45 SNU) assuming the fluxes predicted by the Standard Solar Model of [3]. The procedure of extraction of the daughter cesium atoms from liquid xenon as well as other technical problems concerning preparation of the cesium sample, low background measurements and side reactions for a possible realisation as a solar neutrino detector are discussed. The expected counting rate according to the SSM of [3] for a ${ }^{131} \mathrm{Xe}$ detector is $\approx 1500$ events/y·kton. The results of such a detector will be important for solving the puzzle of the possible existence of vacuum oscillations or the MSW effect and/or input parameters of the Standard Solar Models.
\end{abstract}

\section{Introduction}

Reconciling the Kamiokande and Homestake experiments one was led to the the conclusion that not only ${ }^{8} \mathrm{~B}$ but ${ }^{7} \mathrm{Be}$ neutrinos suffer considerable reduction with respect to predictions of the SSM. The GALLEX [1] and SAGE [2] results support this conclusion. In particular, the SSM prediction for the gallium experiment is $113-132 \mathrm{SNU}$ [3], [⿴囗十, [5]. Since the pp neutrino flux is almost model independent and closely tied with the solar luminosity it was supposed that its contribution to the GALLEX result can be predicted with reasonable precision to be $70 \mathrm{SNU}$ [3]. The boron neutrino contribution fixed by the Kamiokande data in model independent way is equal to $7 \mathrm{SNU}$. Thus, comparing theoretical and experimental results one can see that in the gallium data there is no room for beryllium neutrinos which are expected to contribute $31-34$ SNU or $25-27 \%$ of the total flux in the SSMs. After calibration of the GALLEX experiment with a ${ }^{51} \mathrm{Cr}$ neutrino source a deficit of ${ }^{7} \mathrm{Be}$ neutrinos was claimed at a $3 \sigma$ confidence level [6]. Efforts to explain the reduction of beryllium neutrinos on the basis of variation of the input parameters of the standard solar models were unsuccessful until now according to [7]. 
Therefore the ${ }^{7}$ Be neutrino deficit should be proved in another experiment with essential sensitivity to beryllium neutrinos.

In fact, the most important parameter of the detector is the relative ratio of the ${ }^{7} \mathrm{Be}$ to $\mathrm{pp}$ neutrino yield, which should be larger than in the ${ }^{71} \mathrm{Ga}$ experiments. The latter means that the threshold of the detector should be low enough to avoid possible effects of reduction of the ${ }^{7}$ Be neutrino capture cross section near the threshold, and high enough to reduce the relative contribution of the pp neutrinos in the total yield.

There is a number of solar neutrino detectors proposed for the near and far future. Two of them, Superkamiokande [8] (in operation now) and SNO [9] (Sudbury Neutrino Observatory which will start data taking in the nearest future) are sensitive to boron neutrinos mainly. Therefore these experiments will give no full solution of the problem. The same conclusion is true for ICARUS [10] (a multipurpose liquid argon drift detector which will operate in the Gran Sasso Underground Laboratory). The HELLAZ detector [11] (2000 $\mathrm{m}^{3}$ helium TPC at 5 bar, $77 \mathrm{~K}$ ) would be sensitive to pp and ${ }^{7} \mathrm{Be}$ neutrinos but is far away from real operation, as many other proposals like HERON [12], ${ }^{81} \mathrm{Br}$ [13], ${ }^{115} \mathrm{In}$ [14] and detectors based on low temperature techniques [15], because all of them are in very early stages of their development.

Only two of the proposals, BOREXINO and an iodine detector, that both would be able to detect ${ }^{7}$ Be neutrinos should be on line relatively soon.

The main goal of the BOREXINO [16] experiment is to measure the flux of ${ }^{7} \mathrm{Be}$ neutrinos via elastic scattering in a 100 tons liquid scintillator target. Within the electron energy window $0.25<E_{e}<0.80 \mathrm{MeV} 80 \%$ of the signal comes from ${ }^{7}$ Be neutrinos leading to a high signal rate of 50 counts per day. It is supposed to use an ultra-pure water, liquid scintillator and selected constructing materials to reduce the background of the detector to the extremely low level of 0.1 counts/day.ton (for a signal/background ratio of 5:1). To date a prototype of the BOREXINO detector with a volume of scintillator of $4.5 \mathrm{~m}^{3}$ was mounted and tested successfully in the Gran Sasso Underground Laboratory [16]. An extremely high purity of the scintillator at the level of $\approx(2-5) \cdot 10^{-16} \mathrm{~g} / \mathrm{g}$ for ${ }^{238} \mathrm{U}$ and ${ }^{232} \mathrm{Th}$ contamination was demonstrated. The background of the prototype detector in the energy region of $250-800 \mathrm{keV}$ was $\approx 1800$ counts/day [16] which is however still much higher than required. Therefore still big efforts and time will be needed to reach the required sensitivity.

An experiment based on the ${ }^{127} \mathrm{I}+\nu \rightarrow{ }^{127} \mathrm{Xe}^{*}+e^{-}$neutrino capture reaction to the $3 / 2^{+}$excited state of ${ }^{127} \mathrm{Xe}$ with the threshold at $\mathrm{E}_{\nu}=0.789 \mathrm{MeV}$ was suggested by Haxton and is in the pilot research stage now [17]. However this project suffers of the strong model dependent theoretical estimation of the total capture rate, ranging between 20 and 135 SNU [18. Also experimental efforts as the measurement of the charge-exchange reaction ${ }^{127} \mathrm{I}(\mathrm{p}, \mathrm{n}){ }^{127} \mathrm{Xe}$, which could not be normalized to known beta transitions, as well as the attempt to fix the GT distribution in an experiment at LAMPF, [19] where the involved neutrinos had high multipolarities due to their somewhat higher energies, were of limited value in this respect. 
Therefore it will be difficult to solve the ${ }^{7}$ Be neutrino problem within a reasonable time scale using present detectors, and a new detector would be desirable. We discuss here the possibility to use ${ }^{131} \mathrm{Xe}$ as radiochemical detector with a threshold at $352 \mathrm{keV}$ 21] and with good sensitivity to ${ }^{7}$ Be neutrinos. This possibility is based first on the excellent properties of xenon which allow to use it as a target in a radiochemical experiment as well as on a reliable prediction of the neutrino capture rate in ${ }^{131} \mathrm{Xe}[20$.

\section{Cross sections and capture rates}

The proposed detection process is based on the neutrino capture reaction $\nu+{ }^{131} \mathrm{Xe} \rightarrow{ }^{131} \mathrm{Cs}+e^{-}$. The low threshold of this reaction allows to record approximately one fifth of the solar neutrino flux from the $p-p$ reaction and both lines from ${ }^{7}$ Be decay.

The reduced transition probability for neutrino capture by the ${ }^{131} \mathrm{Xe}$ g.s. can be derived from electron capture of ${ }^{131} \mathrm{Cs}$. The half-life of ${ }^{131} \mathrm{Cs}$ is $T_{1 / 2}=9.689$ days [21] and $\log \left(f_{E C} t_{E C}\right)=5.53$ (the $f_{E C}$ value is taken from [22]), which gives $\left[B_{F}^{+}+B_{G T}^{+}\right]=0.018$.

The cross section for neutrino capture with energy $E_{\nu}$ is defined as

$\sigma_{\nu}\left(E_{\nu}\right)=\frac{g_{A}^{2} g_{V}^{2}}{\pi c^{3} \hbar^{4}} \sum_{E^{\prime}} p_{e} E_{e} F\left(Z, A, E_{e}\right) B_{G T}\left(E^{\prime}\right)$

with the nuclear excitation energy $E^{\prime}$, the electron momentum $p_{e}$ and energy $E_{e}$, the GamowTeller matrix elements $B_{G T}$ and the Fermi function $F\left(Z, A, E_{e}\right)$ according to [22].

The capture rates predicted for ${ }^{131} \mathrm{Xe}$ by the SSM of [3] for the ground-state to ground-state transition are presented in Table 1 (in raw a). They indicate the minimum neutrino capture rate, based on the experimentally known transition strength. To take into account the contribution of the excited states we have performed pn-QRPA calculations following the procedure given in [23], [24]. The chosen parameter set included the Nilsson potential parameter of Ragnarsson and Sheline [25], the pairing interaction strength fitted to reproduce the energy gap, the nuclear deformation obtained from the mass formulae of Moeller and Nix [26] for the daughter nucleus ${ }^{131} \mathrm{Cs}$ and the strength of the Gamow Teller interaction obtained by a fit of experimental half life values of the isotope series of the daughter nucleus Cs. This parameter set gave the best reproduction of experimental data. The ground state matrix element 0.018 was taken from the experimental half-life of ${ }^{131} \mathrm{Cs}$. The proton separation threshold of $5.47 \mathrm{MeV}$ excitation energy in ${ }^{131}$ Cs was taken from [27].

From the level scheme [28] one can see that four excited states (except the forbidden transition to the $7 / 2^{+}$level) are below the ${ }^{7} \mathrm{Be}$ neutrino capture threshold. Neutrino capture to these states will increase the part of the beryllium neutrinos in the total absorption rate compared to the ground state capture.

The Gamow-Teller strength calculated by pn-QRPA results for beryllium neutrinos in a capture rate of $17.8 \mathrm{SNU}$ which is only slightly larger than capture to the ground-state of ${ }^{131} \mathrm{Cs}$ alone (13.6 SNU, see Table 1). However, the determination of matrix elements for the transitions to 
the low-lying excited states, as needed for pep, ${ }^{7} \mathrm{Be},{ }^{13} \mathrm{~N},{ }^{15} \mathrm{O}$ neutrinos, in nuclear structure calculations, includes considerable uncertainties.

To check the parameters of our model calculations we have evaluated the contribution of these states phenomenologically from the systematics of known beta-decay rates in the vicinity of the mass number 131 . There are some $\beta^{-}$decays of ${ }^{127} \mathrm{Te} \rightarrow{ }^{127} \mathrm{I},{ }^{129} \mathrm{Te} \rightarrow{ }^{129} \mathrm{I},{ }^{133} \mathrm{Xe} \rightarrow{ }^{133} \mathrm{Cs}$, ${ }^{135} \mathrm{Xe} \rightarrow{ }^{135} \mathrm{Cs}$, which are similar to the ${ }^{131} \mathrm{Xe} \rightarrow{ }^{131} \mathrm{Cs}\left(3 / 2^{+}\right)$g.s. $\rightarrow\left(5 / 2^{+}\right)$transition.

For all these cases the transition strength to the first $5 / 2_{1}^{+}$state is systematically several times larger then for the $5 / 2_{2}^{+}$state. The $3 / 2^{+} \rightarrow 3 / 2^{+}$and $3 / 2^{+} \rightarrow 1 / 2^{+}$transitions are considerably weaker compared to the transition to the $5 / 2^{+}$excited level. Consequently, according to these arguments the contribution of the low-lying excited states to the beryllium rate estimated in this way would not exceed $5 \%$ of the ground state rate.

The cross section for boron neutrinos cannot be inferred reliably from beta-decay systematics due to the large increase in the capture cross section for ${ }^{8} \mathrm{~B}$ neutrinos caused by the GT strength to exited states with energies between $2-7 \mathrm{MeV}$ above the ground state of ${ }^{131} \mathrm{Cs}$. The QRPA calculations produce for boron neutrinos a rate of $12.7 \mathrm{SNU}$ which is stable enough to variation of model parameters to an accuracy of 20-30\%. The obtained capture rate for boron neutrinos is comparable with the contribution of boron neutrinos for other heavy nuclei, ${ }^{71} \mathrm{Ga},{ }^{81} \mathrm{Br},{ }^{127} \mathrm{I}$, ${ }^{115}$ In (see Table 1).

We have calculated a total neutrino capture rate for ${ }^{131} \mathrm{Xe}$ of $45.2{ }_{-6}^{+12} \mathrm{SNU}$ assuming the fluxes predicted by the SSM of [3]. The uncertainty of the total capture rate is estimated to be of the order of $25-30 \%$ and has been determined mainly by variations of the parameters in the model calculations for the transitions to the highly exited states of ${ }^{131} \mathrm{Cs}$. The possible (p,n) measurement of the transition strength to excited states may allow to reduce the uncertainty of the total capture rate. It should be mentioned also that the xenon detector can be calibrated with a ${ }^{51} \mathrm{Cr}$ neutrino source, as it was successfully performed for GALLEX [1]. Such a procedure can precisely determine the efficiency of beryllium neutrino detection and will decrease the uncertainty of the contribution of the excited states. All described points lead to the statement that the uncertainties for the proposed xenon detector are clearly smaller then for other detectors suggested to measure beryllium neutrinos.

The predicted integral rate of absorption events (including the contribution of the excited states) for the xenon detector from the main neutrino sources is, for the SSM of [3], $R \simeq 1500$ events/kton $\cdot y$. 


\section{Remarks on a possible detector realisation and the background}

The half-life of ${ }^{131} \mathrm{Cs}\left(t_{1 / 2}=9.688 d\right)$ and the physical properties of xenon allow to build up a detector of neutrino capture events based on the extraction of the reaction products ${ }^{131} \mathrm{Cs}$ from the xenon target and subsequent observation of their decays.

The abundance of ${ }^{131} \mathrm{Xe}$ is as large as $21.18 \%$ and therefore natural xenon may be used as a target. For reasonable dimensions of the vessel the xenon must be kept in liquid form. The density of liquid xenon is $3.06 \mathrm{~g} / \mathrm{cm}^{3}$, the boiling temperature $(165 \mathrm{~K})$ is higher than for nitrogen, thus it is not difficult from a technical point of view to build up a liquid xenon target with a mass of one kiloton. Note that a dewar for 600 tons of liquid argon (i.e. $\approx 1.4$ kton of liquid xenon) was designed for the first stage of the ICARUS experiment [10].

For cesium extraction the considerable difference of chemical properties of xenon and cesium can be exploited. The ionization potential of cesium atoms $(3.8 \mathrm{eV})$ is the lowest among all elements. The $\mathrm{Cs}^{+}$ions formed in the process of neutrino capture will be kept in this state because the ionization potential of xenon atoms is much higher $(12.1 \mathrm{eV})$, therefore there will be no free electrons to recombine with cesium ions. Electrons can be produced by natural radioactivities and cosmic rays but estimations show that such processes will be negligible if the detector will be placed in a proper underground environment and the purity of xenon will be kept on a sufficient level. Xenon is an inert gas which can be purified to the level of better than $1 \mathrm{ppb}$ (residual oxygen less than $0.1 \mathrm{ppb}$ ) [29], 30].

Despite of many still unknown details of the behaviour of the $\mathrm{Cs}^{+}$ions in liquid xenon, the possible procedure of the extraction of the daughters would be developed on the base of the methods described in [31], [32], where the behaviour of $\mathrm{Ba}^{++}$ions in liquid and gaseous xenon was studied (with the aim to detect the daughter products of double beta decay of ${ }^{136} \mathrm{Xe}$ ). In the way as was suggested in [32] one or several electrodes can be placed in the dewar with xenon and biased with high negative potential which supplies an electric field of about $1 \mathrm{kV} / \mathrm{cm}$ in the vessel volume. The drifting mobility of positively charged ions in liquid xenon is of the order of $3 \cdot 10^{-4} \mathrm{~cm}^{2} / \mathrm{V} \cdot \mathrm{s}$ [33], thus the collection time of produced $\mathrm{Cs}^{+}$ions over several meters distance will not exceed half an hour. Collected on the electrode, the $\mathrm{Cs}^{+}$ions will be frozen on the surface and kept there for the whole exposure time. Then the collector electrode will be removed from the dewar (with proper precautions to exclude any possible contamination) to prepare the cesium sample, following the technique which was developed in 34.

The measurement of the electron capture of ${ }^{131} \mathrm{Cs}$ to ${ }^{131} \mathrm{Xe}$ can be performed by means of detection of the Auger electrons and X-rays in a low background counter. Due to the relatively high energies of the $K$ - and $L$-lines in ${ }^{131} \mathrm{Cs}$ decay, which are $35 \mathrm{keV}$ and $5 \mathrm{keV}$, respectively, a possible method of measurement could be developed on the base of Si semiconductor detectors 
and a thin solid ${ }^{131}$ Cs sample introduced between them.

One of the most crucial issues for the detection of neutrino capture events is the background related with side reactions that may produce cesium isotopes decaying by electron capture with life time long enough in order to penetrate to the cesium counter. There are four cesium isotopes, ${ }^{129} \mathrm{Cs},{ }^{130} \mathrm{Cs},{ }^{131} \mathrm{Cs}$ and ${ }^{132} \mathrm{Cs}$, whose half-lives are longer than several seconds. The contribution of ${ }^{130} \mathrm{Cs}$ is limited due to the low abundance of this isotope of $4.1 \%$. Two of them, ${ }^{129} \mathrm{Cs}$ and ${ }^{132} \mathrm{Cs}$ are decaying to exited states of xenon and therefore can be eliminated by means of registration of the $\gamma$-rays. The same is true for ${ }^{134} \mathrm{Cs},{ }^{136} \mathrm{Cs}$ and ${ }^{137} \mathrm{Cs}$, which may be present in xenon or can be produced in spontaneous fission of ${ }^{238} \mathrm{U}$. They decay to excited states of barium producing characteristic gamma- rays and therefore can be eliminated.

Thus mainly ${ }^{131} \mathrm{Cs}$ created in side reactions can produce a substantial background.

Protons appear mainly as products of cosmic muons. The relevant reaction ${ }^{131} \mathrm{Xe}(p, n){ }^{131} \mathrm{Cs}$ is usually a main source of background in solar neutrino experiments. The cross-section of this reaction is presently unknown. The contribution of this reaction can be determined in direct measurement of the reaction products in the way as it was done in the GALLEX experiment. In this case some amount of concentrate solution was exposed to a muon beam at CERN.

Neutrons from natural radioactivity with energies of several MeV may take part in (n, $\gamma$ ) reactions. For these reactions there is no path to ${ }^{131} \mathrm{Cs}$ via ${ }^{130} \mathrm{Xe}(\mathrm{n}, \gamma){ }^{131} \mathrm{Xe}$ followed by beta decay to cesium, since ${ }^{131} \mathrm{Xe}$ is stable. Fast neutrons produced by cosmic muons may induce the reaction ${ }^{131} \mathrm{Xe}(\mathrm{n}, \mathrm{p}){ }^{131} \mathrm{I}$ followed by ${ }^{131} \mathrm{Xe}(\mathrm{p}, \mathrm{n}){ }^{131} \mathrm{Cs}$ but its yield is small compared to reactions with primary cosmic rays protons.

Cesium isotopes can be produced in spontaneous fission of possible ${ }^{238} \mathrm{U}$ impurities. Fortunately the relative yield of ${ }^{131} \mathrm{Cs}$ is quite small, since the cesium isotopes fission products appear with mass numbers mainly above 136 . The xenon purification to a level of $10^{-15} \mathrm{~g} / \mathrm{g}$ for uranium and thorium impurities will reduce the amount of ${ }^{131} \mathrm{Cs}$ to a negligible level.

Alpha particles may take part in the following reactions producing ${ }^{131} \mathrm{Cs}:{ }^{128} \mathrm{Xe}(\alpha, n){ }^{131} \mathrm{Ba}$ (followed by electron capture to $\left.{ }^{131} \mathrm{Cs}\right) ;{ }^{128} \mathrm{Xe}(\alpha, p){ }^{131} \mathrm{Cs}$. Secondary protons from the latter reaction also may produce cesium via $(p, n)$ reactions. The naturally occurring alpha particles from the uranium and thorium series have average energies of several $\mathrm{MeV}$ with maximum at 9.0 MeV which is much smaller than the Coulomb barrier of $17.5 \mathrm{MeV}$. Therefore cross sections of these reactions are strongly suppressed. Alpha particles also appear as spallation products of cosmic muons with energy high enough to drive these reactions. However the muon flux deep underground is very weak (e.g., less than $1 /\left(m^{2} \cdot h\right)$ at the Gran Sasso Laboratory), and since the cross sections are very small (on the order of $10^{-29} \mathrm{~cm}^{2}$ per nucleon) the higher-energy alpha particles arising in the detector volume will not produce more then a few cesium atoms per year.

It should be stressed, however, that all mentioned points concerning the extraction of ${ }^{131}$ Cs ions, preparation and measurement of the sample as well as careful consideration of the background 
Table 1: Capture rates predicted by the standard solar model of [3] when using the GamowTeller strength distribution calculated in this work for ${ }^{131} \mathrm{Xe}$, and capture rates for some other targets from [3], given in SNU (a - capture rates for the ground-state to ground-state transitions; b - capture rates including transitions to excited states).

\begin{tabular}{|c|c|c|c|c|c|c|c|}
\hline Isotope & $p-p$ & рер & ${ }^{7} \mathrm{Be}$ & ${ }^{8} \mathrm{~B}$ & ${ }^{13} \mathrm{~N}$ & ${ }^{15} \mathrm{O}$ & Total \\
\hline$\overline{37} \mathrm{Cl}$ & 0.0 & 0.2 & $\bar{~} 1.1$ & 6.1 & "0.1 & 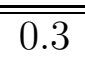 & $\overline{77.9}$ \\
\hline${ }^{40} \mathrm{Ar}$ & 0.0 & 0.0 & 0.0 & 1.7 & 0.0 & 0.0 & 1.7 \\
\hline${ }^{71} \mathrm{Ga}$ & 70.8 & 3.0 & 34.3 & 14.0 & 3.8 & 6.1 & 132.0 \\
\hline${ }^{115} \mathrm{In}$ & 468.0 & 8.1 & 116.0 & 14.4 & 13.6 & 18.5 & 639.0 \\
\hline${ }^{127} \mathrm{I}$ & & 1.85 & 14.0 & 18.4 & 0.727 & 2.43 & 36.4 \\
\hline \multirow{2}{*}{${ }^{131} \mathrm{Xe}$} & 8.7 & 0.9 & 13.6 & 0.5 & 1.1 & 1.3 & 26.1 \\
\hline & 9.7 & 1.6 & 17.8 & 12.7 & 1.6 & 1.8 & 45.2 \\
\hline
\end{tabular}

have to be the subject of additional investigation.

\section{Conclusion}

A solar neutrino problem evidently exists ( in particular for ${ }^{7}$ Be neutrinos). Several possible solutions including "terrestrial", "astrophysical" and "particle physics" solutions were considered. Therefore the ${ }^{7} \mathrm{Be}$ neutrino deficit should be proved by another detector with a better relative ratio of the ${ }^{7} \mathrm{Be}$ to the pp neutrino detection than ${ }^{71} \mathrm{Ga}$ experiments. We have outlined why in addition to the present efforts in this direction, as BOREXINO, a further detector is needed.

In the present work a new solar neutrino detector is proposed which meets this requirement. It is based on neutrino capture in ${ }^{131} \mathrm{Xe}$ with a threshold at $352 \mathrm{keV}$. The total capture rate for this reaction is calculated to be equal to 45.2 SNU. A one kiloton detector will produce a net neutrino signal of $\simeq 1500$ events/yr. The main advantage of the proposed xenon detector is the high sensitivity to ${ }^{7} \mathrm{Be}$ neutrinos, which contribute $\simeq 37 \%$ of the total capture rate.

The uncertainties of the calculated capture rates reflect the contributions from the highly excited states of ${ }^{131} \mathrm{Cs}$ and may be decreased significantly by measuring the transition strength to excited states in $(\mathrm{p}, \mathrm{n})$ reactions and by calibration of the ${ }^{131} \mathrm{Xe}$ detector with a ${ }^{51} \mathrm{Cr}$ neutrino source. Due to the high abundance of ${ }^{131} \mathrm{Xe}(21.18 \%)$ and the physical properties of xenon, there are no technical restrictions to build up the proposed detector with a mass of one kiloton of natural liquid xenon. A procedure of extraction of the daughter cesium atoms from xenon is proposed which is based on collection of $\mathrm{Cs}^{+}$ions on a collector biased with negative potential. The additional research and development for all technical subjects as well as for contributions of side reactions are in progress now.

The feasibility of the proposed xenon detector depends on the particular cost of the xenon. Since the price of xenon in the last years fell down significantly, the cost of large quantities of 
this gas, and even of enriched ${ }^{131} \mathrm{Xe}$, is no longer prohibitive.

The comprehensive analysis of data from the xenon detector together with the results of the gallium, chlorine and Superkamiokande experiments would allow to determine the $p p,{ }^{7} \mathrm{Be}$ and ${ }^{8} \mathrm{~B}$ neutrino fluxes.

This information will be important to constrain solar parameters, or check the existence of vacuum oscillations and MSW effect and finally may help to solve one of the most longstanding puzzles of modern physics.

\section{Acknowledgements}

We thank F.A. Danevich and M. Hirsch for useful discussions. A.Sh.G. and Yu.G.Z. are supported by the Science and Technology Center of Ukraine (contract No. 411).

\section{References}

[1] GALLEX Collab., P. Anselmann et al., Phys. Lett. B 357 (1995) 237;

Proc. Neutrino 96, Helsinki, Finland, June 1996, to be published.

[2] SAGE Collab., J.N. Abdurashitov et al., Phys. Lett. B328 (1994) 234;

Nucl. Phys. B (Proc. Suppl.) 48 (1996) 299.

[3] J.N.Bahcall and M.H. Pinsonneault, Rev. Mod. Phys. 64 (1992) 885; 67 (1995) 781;J.N.Bahcall and R.K.Ulrich, Rev. Mod. Phys. 60 (1988) 297.

[4] S. Turck-Chieze and I. Lopez., Ap. J. 408 (1993) 347.

[5] A. Dar and G. Shaviv, Preprint Technion Ph-94-5 (1994);

G. Shaviv, Nucl. Phys. B (Proc. Suppl.) 38 (1995) 81.

[6] T. Kirsten, Annals of the New York Academy of Sciences, Vol. 759 (1995) 1.

[7] J.N.Bahcall, Nucl. Phys. B (Proc. Suppl.) 48 (1996) 309.

[8] Y. Suzuki, Nucl. Phys. B (Proc. Suppl.) 35 (1994)

Proc. Neutrino 96, Helsinki, Finland, June 1996, to be published.

[9] SNO Collab., M.E. Moorhead, Nucl. Phys. B (Proc. Suppl.) 48 (1996) 378.

[10] C. Rubbia, Nucl. Phys. B (Proc. Suppl.) 48 (1996) 172.

[11] HELLAZ Collab., G. Bonvicini, Nucl. Phys. B 35 (1994) 438.

[12] S.R. Bandler et al., Journal of Low Temp. Phys. 93 (1993) 785;

R.E. Lanou, H.J. Maris, and G.M. Seidel, Phys. Rev. Lett., 58 (1987) 2498;

S.R. Bandler et al., Phys. Rev. Lett. 74 (1995) 3169.

[13] R.D. Scott, Nature 264 (1976) 729;

J.N. Bahcall, Phys. Rev. C 24 (1981) 2216. 
[14] R.S. Raghavan, Phys. Rev. Lett. 37 (1976) 259.

[15] E. Fiorini, Nucl. Phys. B (Proc. Suppl.) 48 (1996) 41.

[16] BOREXINO Collab., G. Bellini, Nucl. Phys. B (Proc. Suppl.) 48 (1996) 363.

[17] W.C. Haxton, Phys. Rev. Lett. 60 (1988) 768.

[18] Yu.S. Lyutostansky and N.B. Shul'gina, Phys. Rev. Lett. 67 (1991) 430.

[19] J. Engel, P.I. Krastev and Lande, Phys. Rev. C 51 (1995) 2837.

[20] A.Sh. Georgadze, H.V. Klapdor-Kleingrothaus, H. Päs, Yu.G. Zdesenko, Astroparticle Physics 7 (1997) 173

[21] Nuclear Data Sheets 72 (1994) 487.

[22] N.B. Gove and M.J. Martin, Nuclear Data Tables 10 (1971) 205.

[23] E. Bender, K. Muto, H.V. Klapdor, Phys. Lett. B 208 (1988) 53;

Z. Phys. A 333 (1989) 125;

K. Muto, E. Bender, T. Oda and H.V. Klapdor-Kleingrothaus, Z. Phys. A 341 (1992) 407.

[24] A. Staudt, E. Bender, K. Muto, H.V. Klapdor-Kleingrothaus, At. Data Nucl. Data Tables $44(1990) 79$.

[25] I. Ragnarsson, R.K. Sheline, Phys. Scr. 29 (1984)385.

[26] P. Moeller, J.R. Nix, At. Data Nucl. Data Tables 39 (1988) 212.

[27] A.H. Wapstra, G. Audi, Nucl. Phys. A 595 (1995) 409. 18

[28] Table of isotopes, ed. by Lederer, C.M., Shirley, V.S. (7-th ed., Wiley, New York, 1978).

[29] P. Belli et al., Nucl. Inst. Meth. in Physics Research A336 (1993) 336.

[30] P. Benetti et al., Nucl. Inst. Meth. in Physics Research A329 (1993) 361.

[31] T.M. Madden, L.W. Mitchell, Nucl. Inst. Meth. in Physics Research A359 (1995) 506.

[32] M.Mijajima et al., IEEE Trans. Nucl. Sci. 41 (1994) 835;

M.Mijajima et al., Proc. RIS-96, 1996, to be published.

[33] H.T. Davis et. al., J. Chem. Phys. 37 (1962) 947.

[34] W.A. Nierenberg et al., Phys. Rev. 112 (1958) 186. 